\title{
Clinical efficacy of atorvastatin calcium combined with aspirin in patients with acute ischemic stroke and effect on neutrophils, lymphocytes and IL-33
}

\author{
WANHUI LI ${ }^{1}$, XIANGYANG REN ${ }^{2}$ and LI ZHANG ${ }^{2}$ \\ Departments of ${ }^{1}$ Rehabilitation and ${ }^{2}$ Neurology, Luoyang Central Hospital \\ Affiliated to Zhengzhou University, Luoyang, Henan 471009, P.R. China
}

Received June 7, 2019; Accepted December 3, 2019

DOI: $10.3892 /$ etm. 2020.8820

\begin{abstract}
Clinical efficacy of atorvastatin calcium combined with aspirin in patients with acute ischemic stroke (AIS) and its effect on neutrophils to lymphocytes ratio (NLR) and interleukin-33 (IL-33) were investigated. In total, 108 patients with AIS in Luoyang Central Hospital Affiliated to Zhengzhou University from April 2016 to October 2017 were selected. There were 56 cases treated with atorvastatin calcium combined with aspirin as the observation group, and 52 cases were treated with aspirin alone as the control group. The clinical effect was observed. The NLR and IL-33 levels were measured by routine blood test and enzyme linked immunosorbent assay (ELISA) before and after treatment. The scores of the National Institutes of Health Stroke scale (NIHSS) and the occurrence of complications were collected before and after treatment in the two groups. Modified Rankin Scale (MRS) was used to evaluate the curative effect. Score $\leq 2$ points is effective in the treatment. Pearson's analysis was used to analyze the correlation between NLR, IL-33 and NIHSS score. The total hospitalization time and 1 year survival rate were compared. The total effective rate of treatment in the observation group was higher than that in the control group $(\mathrm{P}<0.05)$. There was no difference in NLR and IL-33 levels between the two groups before treatment $(\mathrm{P}>0.05)$. After treatment, the NLR in the observation group was significantly lower than that in the control group $(\mathrm{P}<0.05)$. After treatment, the NIHSS score, the total number of complications and the total hospitalization time in the observation group were significantly lower than those in the control group $(\mathrm{P}<0.05)$. Pearson's analysis showed a positive correlation between NLR and NIHSS score $(r=0.681, \mathrm{P}<0.001)$, and a negative correlation
\end{abstract}

Correspondence to: Dr Wanhui Li, Department of Rehabilitation, Luoyang Central Hospital Affiliated to Zhengzhou University, 288 Zhongzhou Middle Road, Luoyang, Henan 471009, P.R. China E-mail:vnhn95@163.com

Key words: atorvastatin calcium, aspirin, combined treatment, acute ischemic stroke, neutrophils to lymphocytes ratio, interleukin-33 between IL-33 and NIHSS score $(r=-0.708, \mathrm{P}<0.001)$. In conclusion, atorvastatin calcium combined with aspirin has a better effective rate in the treatment of acute ischemic stroke than aspirin alone. The combination can better reduce the NLR, increase the expression level of IL-33 in serum, reduce the occurrence of complications and hospitalization time, and increase the survival rate of patients.

\section{Introduction}

Cerebral apoplexy is a serious brain injury disease caused by obstruction of blood circulation in the brain, and its incidence and mortality are much higher than those of other brain injury diseases, which threatens the quality of life and health of many patients; according to statistics, the annual incidence of stroke in China is approximately 116-219 per 100,000 people $(1,2)$. In addition, $85 \%$ of acute stroke belonged to acute ischemic stroke (AIS) (3). AIS has become the leading cause of permanent disability in adults and the second most common cause of dementia, and its mortality rate ranks third in the world (4). According to epidemiological statistics in the United States, 610,000 new cases occur every year, and the incidence is still increasing year by year. The incidence of stroke has increased by $12 \%$ in low and middle income countries over the past 30 years, and the people suffering from stroke have become younger (5-7). According to the condition of the patient, the corresponding treatment plan can be provided, but Zhong et al (8) reported on 2,944 patients in 22 hospitals in Suzhou and found that $3.7 \%$ of the patients died directly during in-hospital treatment. In addition, it was reported that $20 \%$ of AIS patients have cardiemphraxis and $76 \%$ of AIS patients have obvious autonomic nervous dysfunction $(9,10)$. Zhong et al (11) results on 253,680 patients show that after treatment, the patients were admitted back to hospital because of infection, coronary artery disease and recurrent stroke, and the readmission rates for 30 days and 1 year were 17.4 and $42.5 \%$ respectively.

AIS causes strong inflammatory reaction, so some inflammatory indexes are closely related to AIS process (12). The neutrophil-to-lymphocyte ratio (NLR) is an inflammatory predictor widely used in the diagnosis of cancer (13). Some 
studies have also found that NLR can be used to predict the risk of cardiovascular and cerebrovascular diseases, and to predict the early clinical results of AIS $(14,15)$. Interleukin (IL-33) is a member of the IL-1 family and binds to its receptor ST2 to prevent hypertrophy and fibrosis in the myocardium (16). The low level of serum IL-33 is associated with the large infarct volume and greater stroke severity of the AIS patient, and IL-33 can be used as a biomarker for diagnosis and for predicting the prognosis (17).

In order to prevent obstruction, patients generally need to use aspirin that can inhibit platelet aggregation (18). Atorvastatin is a drug with lipid-regulating effect, and is used for treating cardiovascular and cerebrovascular diseases such as hypercholesterolemia and coronary heart disease. Recent studies have reported that atorvastatin can also play a beneficial role in cerebral circulation and cerebral parenchyma during ischemic stroke and reperfusion, which can protect the nerves of patients with AIS. The levels of tumor necrosis factor- $\alpha$, interleukin (IL)- 6 and vascular cell adhesion molecule-1 in patient's plasma were significantly decreased by taking atorvastatin (19). The study of Pignatelli et al (20) shows that atorvastatin can rapidly reduce oxidative stress and platelet activation by directly inhibiting platelet NOx2, and finally inhibiting platelet isoprostol and thrombus A2. Aspirin has been used to treat AIS patients with anti-blocking therapy, but the specific efficacy of aspirin combined with atorvastatin and its effect on NLR and IL-33 were not clear.

Therefore, we used atorvastatin combined with aspirin in the treatment of AIS patients and observe its clinical efficacy and the effect on NLR and IL-33, so as to provide evidence and direction for clinical treatment.

\section{Patients and methods}

General patient data. This is a retrospective study. Altogether 108 patients with AIS treated in Luoyang Central Hospital Affiliated to Zhengzhou University (Luoyang, China) from April 2016 to October 2017 were selected as the subject. The patients were divided into groups according to their medical records as archived by the Luoyang Central Hospital. The 56 patients in the observation group were treated with atorvastatin combined with aspirin, and included 37 males and 19 females, with an average age of $51.63 \pm 9.41$ years. Further 52 patients were treated with aspirin alone as the control group in this study, including 40 males and 12 females, with an average age of $52.46 \pm 10.54$ years. This study was approved by the Medical Ethics Committee of Luoyang Central Hospital Affiliated to Zhengzhou University, and all the patients were informed and signed the informed consent form.

Inclusion and exclusion criteria. Inclusion criteria: the patient was diagnosed with AIS by imaging and pathology; the diagnostic criteria were in line with the guidelines issued by the Stroke Committee of the American Heart Association in 2013 (21); all the patients were admitted to hospital within $6 \mathrm{~h}$ of onset; patients with complete clinical data; patients could be followed up by telephone.

Exclusion criteria: patients with severe liver and renal insufficiency; patients with other malignant tumors; patients with serious cardiovascular and cerebrovascular diseases; patients with severe inflammation; pregnant or lactating women.

Instruments and kits. Blood analyzer (SYSMEX, XS-800i) and its matching reagent were used for detection of blood routine indexes. IL-33 enzyme-linked immunosorbent assay (ELISA) detection kit (Shanghai Enzyme-linked Biotechnology Co., Ltd., ml058087), aspirin (Shandong Xinhua Pharmaceutical Co., Ltd., SFDA approval no. H37020354), atorvastatin (Pfizer, SFDA approval no. H20051407).

Therapeutic regimen. Both groups were treated with routine treatment such as anti-infection and prevention of stress ulcer after admission. The control group was given $100 \mathrm{mg}$ of oral aspirin once a day on the basis of the routine treatment. The observation group was treated with $10 \mathrm{mg}$ of oral atorvastatin once a day on the basis of the treatment of the control group.

Sample collection. Aseptic venous blood $(8 \mathrm{ml})$ was collected at 7:00 a.m. the next day after admission; $3 \mathrm{ml}$ was added to the anticoagulant tube, and $5 \mathrm{ml}$ was added to the coagulation tube. The NLR expression in venous blood of anticoagulant tube was detected by blood routine examination. The venous blood in the coagulation tube was centrifuged immediately at $3,000 \mathrm{x} \mathrm{g}$ at $4^{\circ} \mathrm{C}$ for $10 \mathrm{~min}$. The serum was then separated and placed in a refrigerator at $-80^{\circ} \mathrm{C}$.

ELISA detection method. IL-33 was detected by ELISA. The sample was diluted with sample diluent at 1:1, and then $50 \mu \mathrm{l}$ of the diluted sample was added to the reaction well. Then, $50 \mu \mathrm{l}$ of diluted standard material or $50 \mu \mathrm{l}$ of sample to be tested was added into reaction well or blank well. Immediately $50 \mu \mathrm{l}$ of biotin labeled antibody was added. The well was covered with the membrane plate and the sample was gently shaken and mixed and incubated at $37^{\circ} \mathrm{C}$ for $1 \mathrm{~h}$. Then the liquid in the well was discarded and each reaction well was filled with washing fluid. Then shaken for $30 \mathrm{sec}$, discarding the washing fluid, and dried with absorbent paper. This operation was repeated 3 times. Then $80 \mu 1$ of affinity enzyme-HRP was added to each well. The sample was gently shaken and mixed and incubated at $37^{\circ} \mathrm{C}$ for $30 \mathrm{~min}$. The liquid in the well was discarded and each reaction well was filled with washing fluid. Then shaken for $30 \mathrm{sec}$, discarding the washing fluid, and dried with absorbent paper. This stage was also repeated 3 times. Then $50 \mu \mathrm{l}$ of substrate A and $50 \mu \mathrm{l}$ of B were added to each well. The sample was gently shaken and mixed and incubated at $37^{\circ} \mathrm{C}$ for $10 \mathrm{~min}$ avoid light. The enzyme standard plate was taken out and $50 \mu 1$ of terminating solution was added quickly. The results were determined immediately after the termination solution was added. The optical density value (OD value) of each well was detected at the wavelength of $450 \mathrm{~nm}$.

Follow-up. A total of 108 patients or family members were followed up by telephone and interview bimonthly. The follow-up time was 1 year.

Observation index. Main observation index: the NLR and IL-33 levels in observation group and control group before 
Table I. Clinical data of patients $[\mathrm{n}(\%)$, mean \pm SD].

\begin{tabular}{|c|c|c|c|c|}
\hline Factors & Observation group $(n=56)$ & Control group $(\mathrm{n}=52)$ & $\mathrm{t} / \chi^{2} / \mathrm{Z}$ value & P-value \\
\hline Sex & & & 1.552 & 0.213 \\
\hline Male & $37(66.07)$ & $40(76.92)$ & & \\
\hline Female & $19(33.93)$ & $12(23.08)$ & & \\
\hline Age (years) & $51.63 \pm 9.41$ & $52.46 \pm 10.54$ & 0.432 & 0.666 \\
\hline $\mathrm{BMI}\left(\mathrm{kg} / \mathrm{m}^{2}\right)$ & $23.65 \pm 1.82$ & $24.04 \pm 1.97$ & 1.069 & 0.287 \\
\hline \multicolumn{5}{|l|}{ Previous medical history } \\
\hline Hypertension & $19(33.93)$ & $21(40.38)$ & 0.482 & 0.488 \\
\hline Diabetes & $13(23.21)$ & $10(19.23)$ & 0.255 & 0.613 \\
\hline Hyperlipidemia & $8(14.29)$ & $7(13.46)$ & 0.015 & 0.902 \\
\hline Smoking history & & & 0.097 & 0.755 \\
\hline Yes & $21(37.50)$ & $18(34.62)$ & & \\
\hline No & $35(62.50)$ & $34(65.38)$ & & \\
\hline Alcohol abuse history & & & 0.186 & 0.667 \\
\hline Yes & $9(16.07)$ & $10(19.23)$ & & \\
\hline No & $47(83.93)$ & $42(80.77)$ & & \\
\hline Residence & & & 1.055 & 0.304 \\
\hline Urban & $43(76.79)$ & $44(84.62)$ & & \\
\hline Rural & $13(23.21)$ & $8(15.38)$ & & \\
\hline Platelet count (x109/1) & $152.93 \pm 54.41$ & $147.23 \pm 51.62$ & 0.558 & 0.578 \\
\hline Blood glucose (mmol/l) & $6.67 \pm 2.21$ & $6.52 \pm 1.86$ & 0.380 & 0.705 \\
\hline Urea nitrogen (mmol/l) & $6.17 \pm 3.72$ & $6.29 \pm 3.81$ & 0.166 & 0.869 \\
\hline Creatine $(\mu \mathrm{mol} / \mathrm{l})$ & $75.86 \pm 13.74$ & $77.29 \pm 15.31$ & 0.512 & 0.610 \\
\hline $\mathrm{cTnT}(\mu \mathrm{g} / \mathrm{l})$ & $5.32 \pm 2.47$ & $5.36 \pm 2.51$ & 0.269 & 0.789 \\
\hline $\mathrm{cTnI}(\mu \mathrm{g} / \mathrm{l})$ & $8.43 \pm 1.66$ & $8.29 \pm 1.54$ & 0.453 & 0.651 \\
\hline Total cholesterol (mmol/l) & $6.58 \pm 0.87$ & $6.54 \pm 0.83$ & 0.244 & 0.808 \\
\hline Glycerin trilaurate $(\mathrm{mmol} / \mathrm{l})$ & $3.32 \pm 0.74$ & $3.47 \pm 0.81$ & 1.006 & 0.317 \\
\hline Infarct location & & & 0.537 & 0.999 \\
\hline Frontal lobe & $9(16.07)$ & $9(17.31)$ & & \\
\hline Temporal lobe & $7(12.50)$ & $7(13.46)$ & & \\
\hline Parietal lobe & $6(10.71)$ & $5(9.62)$ & & \\
\hline Occipital lobe & $6(10.71)$ & $7(13.46)$ & & \\
\hline Basal ganglion & $8(14.29)$ & $7(13.46)$ & & \\
\hline Thalamus & $9(16.07)$ & $7(13.46)$ & & \\
\hline Cerebellum & $8(14.29)$ & $8(15.38)$ & & \\
\hline Brainstem & $3(5.36)$ & $2(3.85)$ & & \\
\hline Infarct size & & & 0.248 & 0.804 \\
\hline Lacunar infarction & $32(57.14)$ & $28(53.85)$ & & \\
\hline Medium infarction & $16(28.57)$ & $17(32.69)$ & & \\
\hline Massive infarction & $8(14.29)$ & $7(13.46)$ & & \\
\hline
\end{tabular}

and after treatment were compared; the scores of National Institutes of Health Stroke scale (NIHSS) before and after treatment were compared; the Modified Rankin Scale (MRS) was used for evaluation of curative effect (score equal to or less than 2 points is effective in the treatment), total effective rate of treatment, and survival rate of patients 1 year after treatment.
Secondary observation index: clinical data of the two groups of patients, total length of hospitalization, the occurrence of complications.

Statistical methods. SPSS20.0 (SPSS) medical statistical analysis software was used to statistically analyze the collected data, and GraphPad Prism 7 (GraphPad) to plot figures. 

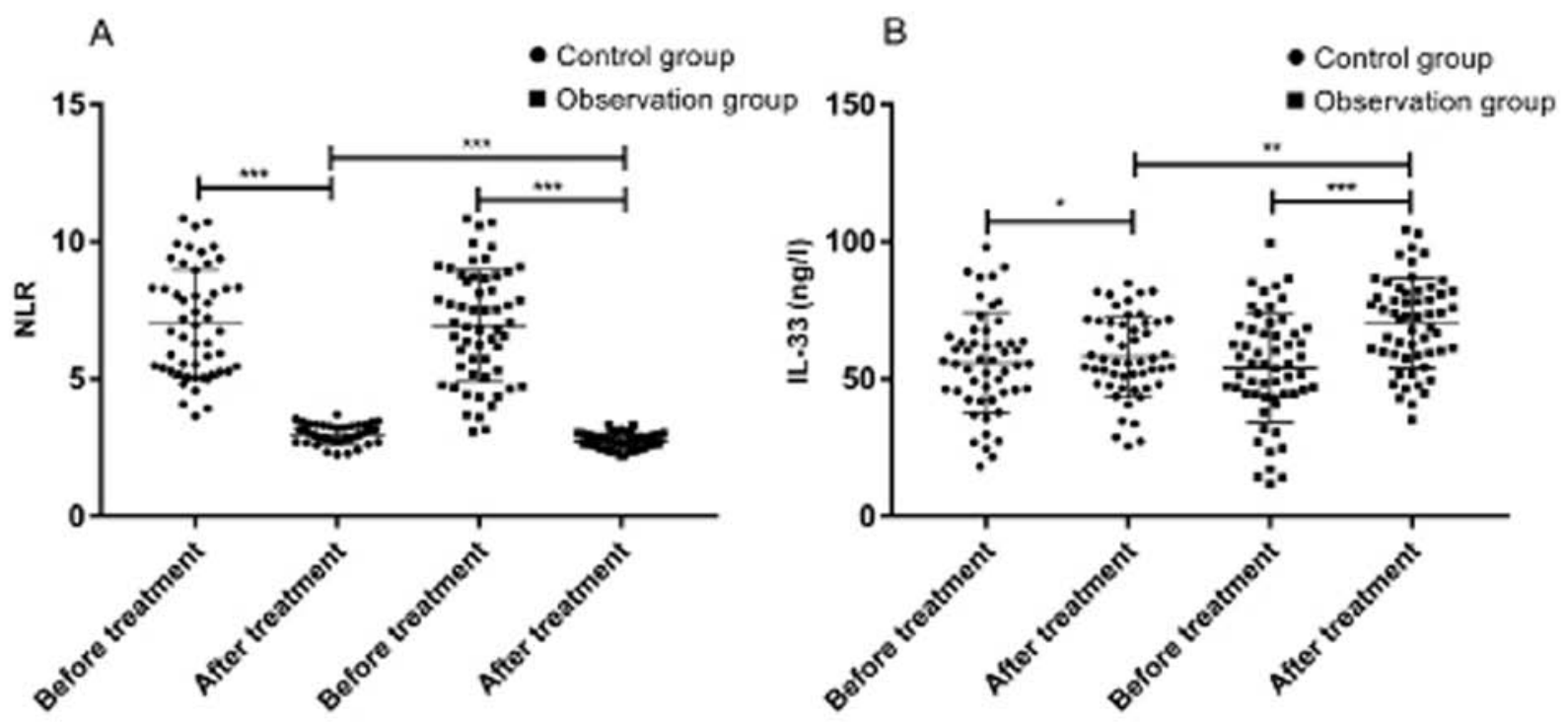

Figure 1. NLR ratio and IL-33 level before and after treatment in the two groups. (A) There was no significant difference in NLR between the two groups before treatment $(\mathrm{t}=0.192, \mathrm{P}=0.849)$. After treatment, the NLR of the control group was significantly lower than that before treatment $(\mathrm{t}=14.689, \mathrm{P}<0.001)$; $\mathrm{NLR}$ was significantly lower in the observation group than before treatment $(t=15.401, \mathrm{P}<0.001)$, and the NLR in the observation group was significantly lower than that in the control group $(\mathrm{t}=8.961, \mathrm{P}<0.001)$. (B) There was no significant difference in IL-33 between the two groups before treatment $(\mathrm{t}=0.540, \mathrm{P}=0.590)$. After treatment, IL-33 was significantly higher in the control group than before treatment $(\mathrm{t}=2.440, \mathrm{P}=0.020)$; IL-33 was significantly higher than before treatment $(\mathrm{t}=4.336, \mathrm{P}<0.001)$, and IL-33 was significantly higher in the observation group than in the control group $(\mathrm{t}=2.910, \mathrm{P}=0.004) .{ }^{*} \mathrm{P}<0.05,{ }^{* *} \mathrm{P}<0.01,{ }^{* * *} \mathrm{P}<0.001$. NLR, neutrophils to lymphocytes ratio; IL-33, interleukin-33.

Table II. NIHSS scores of patients in both groups before and after treatment (mean \pm SD).

\begin{tabular}{|c|c|c|c|c|}
\hline Treatment & Observation group $(\mathrm{n}=56)$ & Control group $(n=52)$ & $\mathrm{t}$ value & P-value \\
\hline Before treatment & $11.36 \pm 3.74$ & $11.41 \pm 3.78$ & 0.234 & 0.816 \\
\hline After treatment & $6.75 \pm 1.28^{\mathrm{a}}$ & $7.86 \pm 2.34^{\mathrm{a}}$ & 3.088 & 0.003 \\
\hline Difference & $4.62 \pm 1.35$ & $3.53 \pm 1.16$ & 4.483 & $<0.001$ \\
\hline
\end{tabular}

${ }^{a} \mathrm{P}<0.05$ indicates that there is a significant difference between after treatment and before treatment. NIHSS, National Institutes of Health Stroke scale.

Enumeration data utilization rate $(\%)$ was detected by Chi-square test and represented by $\chi^{2}$. Fisher's test was used when the number of samples was $\geq 40$, and the theoretical frequency was $<1$. The measurement data were represented by mean \pm standard deviation (mean $\pm \mathrm{SD}$ ), and all measurement data were in accordance with normal distribution. Independent sample t-test was used for comparison between the two groups. Intra-group comparison used pairing sample t-test and was represented by t. Grade data were analyzed using rank sum test, and Pearson's analysis was used to analyze the correlation between NLR, IL-33 and NIHSS score. K-M survival was used for analysis of 1 year survival of patients. The log rank test was used for analysis. $\mathrm{P}<0.05$ was considered to indicate a statistically significant difference.

\section{Results}

General data of the two groups. The clinical data of the two groups were collected and compared, the results showed that there was no significant difference in sex, age, BMI, previous medical history (hypertension, diabetes, hyperlipidemia), smoking history, alcohol abuse history, residence, platelet count, blood glucose, urea nitrogen, creatine, cardiac troponin $\mathrm{T}$ (cTnT), cardiac troponin I (cTnI), total cholesterol, glycerin trilaurate, infarct location and infarct size between the observation group and the control group $(\mathrm{P}>0.05)$ (Table I).

Comparison of NLR and IL-33 level between the two groups before and after treatment. The NLR in the two groups before and after treatment was compared, but there was no difference between the observation group $(6.58 \pm 2.14)$ and the control group $(6.64 \pm 2.20)$ before treatment. The NLR of the observation group (2.74 \pm 0.16$)$ and the control group (3.07 \pm 0.22$)$ after treatment was significantly lower than that before treatment $(\mathrm{P}<0.05)$. The NLR of the observation group after treatment was significantly lower than that of the control group $(\mathrm{P}<0.05)$, and the difference was significantly higher than that of the control group $(\mathrm{P}<0.05)$. The level of IL-33 in the two groups before and after treatment was compared. There was no difference between 

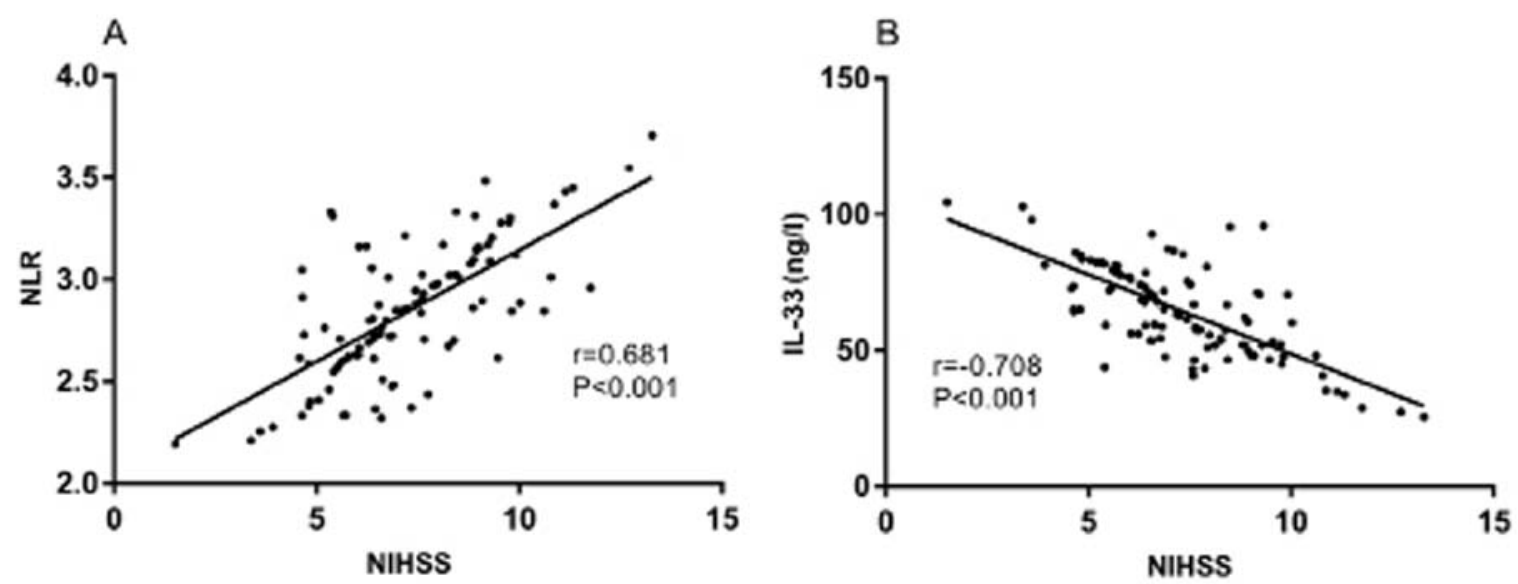

Figure 2. Correlation between NLR, IL-33 and NIHSS score. (A) NIHSS score was positively correlated with NLR expression ( $r=0.681$, P $<0.001$ ). (B) NIHSS score was negatively correlated with IL-33 expression ( $r=-0.708, P<0.001)$. NLR, neutrophils to lymphocytes ratio; IL-33, interleukin-33; NIHSS, National Institutes of Health Stroke scale.

Table III. Complications of the two groups [n (\%)].

\begin{tabular}{lcccr}
\hline Complication & Observation group $(\mathrm{n}=56)$ & Control group $(\mathrm{n}=52)$ & $\chi^{2}$ value & P-value \\
\hline Gastrointestinal bleeding & $2(3.57)$ & $3(5.77)$ & 0.295 & 0.587 \\
Heart rate disorder & $7(12.50)$ & $15(28.85)$ & 4.441 & 0.035 \\
Pulmonary infection & $4(7.14)$ & $6(11.54)$ & 0.620 & 0.431 \\
Epilepsia & $4(7.14)$ & $8(15.38)$ & 1.854 & 0.173 \\
Brain edema & $6(10.71)$ & $13(25.00)$ & 3.796 & 0.051 \\
\hline
\end{tabular}

the observation group $(54.28 \pm 18.24 \mathrm{ng} / \mathrm{l})$ and the control group $(52.47 \pm 16.46 \mathrm{ng} / \mathrm{l})$ before treatment. After treatment, The level of IL-33 in the two groups was significantly higher than that before treatment $(\mathrm{P}<0.05)$. The level of IL-33 in the observation group $(68.86 \pm 15.46 \mathrm{ng} / \mathrm{l})$ was significantly higher than that in the control group $(59.27 \pm 18.74 \mathrm{ng} / \mathrm{l})$ after treatment $(\mathrm{P}<0.05)$, and the difference was significantly higher than that in the control group $(\mathrm{P}<0.05)$ (Fig. 1).

Comparison of NIHSS score between the two groups before and after treatment. The NIHSS scores of the two groups before and after treatment were compared, and there was no difference between the two groups $(\mathrm{P}>0.05)$. The NIHSS scores in both groups after treatment were significantly lower than those before treatment $(\mathrm{P}<0.05)$. The NIHSS score in the observation group was significantly lower than that in the control group $(\mathrm{P}<0.05)$, and the difference was significantly higher than that in the control group $(\mathrm{P}<0.05)$ (Table II).

Correlation between NLR, IL-33 and NIHSS score. The relationship between NLR, IL-33 and NIHSS scores was analyzed by Pearson's correlation analysis. NLR was positively correlated with NIHSS score, and IL-33 was negatively correlated with NIHSS score (Fig. 2).

Complications in both groups. By comparing the complications between the two groups, it was found that there was no significant difference in gastrointestinal bleeding, pulmonary infection and epilepsia between the observation group and the control group $(\mathrm{P}>0.05)$, but the heart rate and brain edema in the observation group were significantly lower than those in the control group $(\mathrm{P}<0.05)$ (Table III).

Evaluation of curative effect in the two groups. By comparing the curative effect evaluation of the two groups, it was found that the total effective rate of the patients in the observation group was much higher than that in the control group $(\mathrm{P}<0.05)$ (Table IV).

Comparison of the total hospitalization time between the two groups. By comparing the total hospitalization time of the two groups, it was found that the total hospitalization time of the patients in the observation group $(9.24 \pm 3.42$ days) was significantly shorter than that in the control group (11.57 \pm 4.78 days) $(\mathrm{P}<0.05)$ (Fig. 3).

One year survival rate of the two groups. According to the statistics of one year survival of the two groups, 108 patients or family members were followed up; 0 patients were lost; 29 patients died; 79 survived at one year, and the survival rate was $73.15 \%$. In the observation group, 10 cases died, 46 cases survived, the survival rate was $82.14 \%$. In the control group, 19 cases died, 33 cases survived, the survival rate was $63.46 \%$. By drawing the one year survival rate of the two groups, it was found that the one year survival rate of the patients in 
Table IV. Evaluation of the curative effect of the two groups of patients [n (\%)].

\begin{tabular}{lccrr}
\hline MRS & Observation group $(\mathrm{n}=56)$ & Control group $(\mathrm{n}=52)$ & $\chi^{2}$ value & P-value \\
\hline 0 No symptoms & $19(33.93)$ & $12(23.08)$ & 1.552 & 0.213 \\
1 Symptomatic, no significant disability & $18(32.14)$ & $11(21.15)$ & 1.658 & 0.198 \\
2 Slight disability & $9(16.07)$ & $8(15.38)$ & 0.010 & 0.922 \\
3 Moderate disability & $6(10.71)$ & $9(17.31)$ & 0.980 & 0.322 \\
4 Moderately severe disability & $2(3.57)$ & $7(13.45)$ & 3.453 & 0.063 \\
5 Severe disability & $2(3.57)$ & $5(9.62)$ & 1.625 & 0.202 \\
6 Dead & $0(0.00)$ & $0(0.00)$ & & $>0.999$ \\
Total effective treatment & $46(82.14)$ & $31(59.62)$ & 6.686 & 0.010 \\
\hline
\end{tabular}

MRS, Modified Rankin Scale.

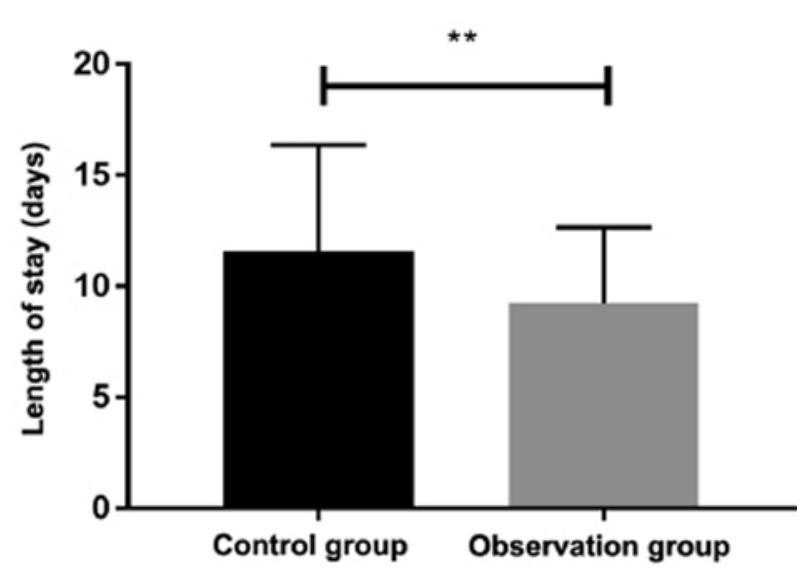

Figure 3. Comparison of total postoperative hospitalization time between the two groups. The total hospitalization time in the observation group was shorter than that in the control group, and there was a significant difference $(\mathrm{t}=2.770, \mathrm{P}=0.004) .{ }^{* *} \mathrm{P}<0.01$.

the observation group was much better than that in the control group ( $\mathrm{P}=0.017)$ (Fig. 4).

\section{Discussion}

In this study, we found that the NLR of AIS patients treated with atorvastatin combined with aspirin and those treated with aspirin alone were significantly lower than those before treatment. We speculate that with the development of therapeutic efficacy, the inflammation of patients is reduced, so NLR, an inflammatory marker, also decreases. In the study of Hao et al (22), interventional therapy combined with routine drug therapy and single drug therapy reduced the loss of nerve function in patients with ischemic cerebrovascular disease, and NLR decreased significantly with the treatment and improvement of patients. The NLR in the observation group was significantly lower than that in the control group after treatment, which may suggest that the combination therapy is better than aspirin alone to reduce inflammation. The level of IL-33 in both groups was significantly higher than that in the control group after treatment. In the study of Yang et al (23), it is mentioned that the infusion of IL-33 into rats with transient middle cerebral artery occlusion can reduce the area

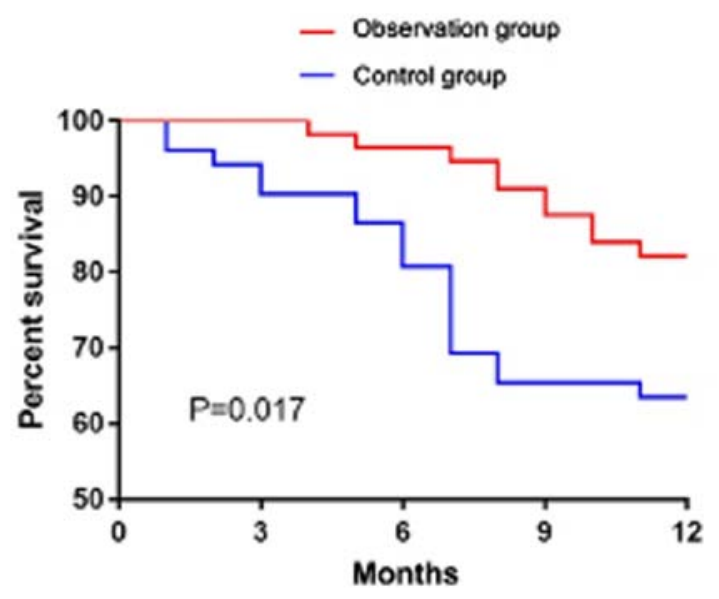

Figure 4. One year survival of patients in both groups. The one year survival rate was $82.14 \%$ in the observation group and $63.46 \%$ in the control group. The one year survival rate in the observation group was significantly better than that in the control group $(\mathrm{P}=0.017)$.

of cerebral infarction, while IL-33 enhances the expression of IL-10, anti-inflammatory and tissue repair M2 genes in primary microglia, enhances the survival of neurons, and play a neuroprotective role. Panahi et al (24) also report that IL-33 converts microglia from inflammatory M1 to anti-inflammatory and tissue repair M2 phenotypes to reduce brain injury caused by ischemic stroke. Therefore, we speculate that after treatment, the level of IL-33 increases to protect and repair the nerve, and the patient's anti-inflammation and repair function are enhanced, so that the condition can be improved. After treatment, the level of IL-33 in the observation group was significantly higher than that in the control group, suggesting that the patients treated with combined medication may have more improvement and stronger ability of anti-inflammation and tissue repair than those treated with aspirin alone. This result is also similar to the results of Galun et al (25), finding that stroke patients with smaller cerebral infarction had higher serum IL-33, and mild stroke patients had higher serum IL-33 than severe stroke patients.

The specific pathogenesis of AIS is not clear, but inflammation and thrombosis are currently considered to be the key factors in the pathogenesis of ischemic stroke $(17,26,27)$. 
Platelet activation and coagulation thrombosis play an important role in the pathological and physiological process of recurrent ischemic vascular events in stroke patients (28). Aspirin is a conventional antiplatelet drug, but in recent years, it was reported that platelet aggregation in patients with AIS treated with statins combined with aspirin is significantly lower than that in patients without treatment, and the incidence of neurological deterioration was less than that of patients without treatment (29). Therefore, we studied the efficacy of atorvastatin combined with aspirin compared with aspirin alone.

The NIHSS scores of the two groups before and after treatment were compared. NIHSS score can be used to assess stroke severity (30). It was found that the NIHSS score of the two groups after treatment was significantly lower than that before treatment, and the score of the observation group was significantly lower than that of the control group, which indicated that the condition of the patients was improved after drug treatment, and the improvement degree of the patients in the observation group was better than that in the control group. Pearson's correlation analysis was used to detect the correlation between NLR and IL-33 and NIHSS scores. NLR was positively correlated with NIHSS score, and IL-33 was negatively correlated with NIHSS score. Then, the complications between the two groups was compared. There was no significant difference in gastrointestinal bleeding, pulmonary infection and epilepsy between the observation group and the control group. The number of heart rate disorders and brain edema and the total number of complications in the observation group were significantly lower than those in the control group. The curative effect of the two groups was evaluated by MRS score, and it was found that the total effective rate of the patients in the observation group was significantly higher than that in the control group, while the total hospitalization time in the observation group was significantly lower than that in the control group. Finally, the 1-year survival rate of all patients was studied, and it was found that the 1-year survival rate of all patients was $73.13 \%$; that of the observation group was $82.14 \%$, and that of the control group was $63.46 \%$. The 1-year survival rate of the observation group was significantly higher than that of the control group. This suggests that atorvastatin combined with aspirin can improve the survival of patients.

Collectively, atorvastatin calcium combined with aspirin has a better effective rate in the treatment of acute ischemic stroke than aspirin alone. The combination can reduce the NLR, increase the expression level of IL-33 in serum, reduce the occurrence of complications and hospitalization time, and increase the survival rate of patients.

\section{Acknowledgements}

Not applicable.

\section{Funding}

No funding was received.

\section{Availability of data and materials}

The datasets used and/or analyzed during the present study are available from the corresponding author on reasonable request.

\section{Authors' contributions}

WL and XR were responsible for ELISA. LZ analyzed and interpreted the patient data. XR helped with statistical analysis. WL wrote the manuscript. All authors read and approved the final manuscript.

\section{Ethics approval and consent to participate}

The study was approved by the Medical Ethics Committee of Luoyang Central Hospital Affiliated to Zhengzhou University (Luoyang, China). Signed informed consents were obtained from the patients and/or the guardians.

\section{Patient consent for publication}

Not applicable.

\section{Competing interests}

The authors declare that they have no competing interests.

\section{References}

1. Wang J, Hu Z, Yang S, Liu C, Yang H, Wang D and Guo F: Inflammatory cytokines and cells are potential markers for patients with cerebral apoplexy in intensive care unit. Exp Ther Med 16: 1014-1020, 2018.

2. Suwanwela NC, Poungvarin N and Asap; Asian Stroke Advisory Panel: Stroke burden and stroke care system in Asia. Neurol India 64 (Suppl): S46-S51, 2016.

3. Haq S, Mathur M, Singh J, Kaur N, Sibia RS and Badhan R: Colour Doppler evaluation of extracranial carotid artery in patients presenting with acute ischemic stroke and correlation with various risk factors. J Clin Diagn Res 11: TC01-TC05, 2017.

4. Goldstein LB, Bushnell CD, Adams RJ, Appel LJ, Braun LT, Chaturvedi S, Creager MA, Culebras A, Eckel RH, Hart RG, et al; American Heart Association Stroke Council; Council on Cardiovascular Nursing; Council on Epidemiology and Prevention; Council for High Blood Pressure Research; Council on Peripheral Vascular Disease, and Interdisciplinary Council on Quality of Care and Outcomes Research: Guidelines for the primary prevention of stroke: A guideline for healthcare professionals from the American Heart Association/American Stroke Association. Stroke 42: 517-584, 2011.

5. Bonardo P, Pantiu F, Chertcoff A, León Cejas L, Pacha S, Uribe Roca C, Ernst G, Fernández Pardal M and Reisin R: Blood pressure evolution in young patients with acute ischemic stroke: A new model for understanding the natural course of spontaneous hypertension? Int J Neurosci 128: 140-145, 2018.

6. Jardim PC, Souza WK, Lopes RD, Brandão AA, Malachias MV, Gomes MM, Moreno Júnior H, Barbosa EC and Póvoa RM: I RBH - First Brazilian Hypertension Registry. Arq Bras Cardiol 107: 93-98, 2016.

7. Owaki A, Inaguma D, Aoyama I, Inaba S, Koide S, Ito E, Takahashi K, Hayashi H, Hasegawa M and Yuzawa Y; AICOPP group: Serum phosphate level at initiation of dialysis is associated with all-cause mortality: A multicenter prospective cohort study. Ren Fail 40: 475-482, 2018.

8. Zhong C, You S, Chen J, Zhai G, Du H, Luo Y, Dong X, Cao Y, Liu CF and Zhang Y: Serum alkaline phosphatase, phosphate, and in-hospital mortality in acute ischemic stroke patients. J Stroke Cerebrovasc Dis 27: 257-266, 2018.

9. Wrigley P, Khoury J, Eckerle B, Alwell K, Moomaw CJ, Woo D, Flaherty ML, De Los Rios la Rosa F, Mackey J, Adeoye O, et al: Prevalence of positive troponin and echocardiogram findings and association with mortality in acute ischemic stroke. Stroke 48: 1226-1232, 2017.

10. Xiong L, Tian G, Leung H, Soo YOY, Chen X, Ip VHL, Mok VCT, Chu WCW, Wong KS and Leung TWH: Autonomic dysfunction predicts clinical outcomes after acute ischemic stroke: A prospective observational study. Stroke 49: 215-218, 2018. 
11. Zhong W, Geng N, Wang P, Li Z and Cao L: Prevalence, causes and risk factors of hospital readmissions after acute stroke and transient ischemic attack: A systematic review and meta-analysis. Neurol Sci 37: 1195-1202, 2016.

12. Luo WJ and Zhang WF: The relationship of blood cell-associated inflammatory indices and diabetic retinopathy: A Meta-analysis and systematic review. Int J Ophthalmol 12: 312-323, 2019.

13. Lian L, Xia YY, Zhou C, Shen XM, Li XL, Han SG, Zheng Y, Mao ZQ, Gong FR, Wu MY, et al: Application of platelet/ lymphocyte and neutrophil/lymphocyte ratios in early diagnosis and prognostic prediction in patients with resectable gastric cancer. Cancer Biomark 15: 899-907, 2015.

14. Wu M, Zhou L, Zhu D, Lai T, Chen Z and Shen H: Hematological indices as simple, inexpensive and practical severity markers of obstructive sleep apnea syndrome: A meta-analysis. J Thorac Dis 10: 6509-6521, 2018.

15. Yu S, Arima H, Bertmar C, Clarke S, Herkes G and Krause M: Neutrophil to lymphocyte ratio and early clinical outcomes in patients with acute ischemic stroke. J Neurol Sci 387: 115-118, 2018.

16. Chen WY, Hong J, Gannon J, Kakkar R and Lee RT: Myocardial pressure overload induces systemic inflammation through endothelial cell IL-33. Proc Natl Acad Sci USA 112: 7249-7254, 2015.

17. Qian L, Yuanshao L, Wensi H, Yulei Z, Xiaoli C, Brian W, Wanli Z, Zhengyi C, Jie X, Wenhui Z, et al: Serum IL-33 is a novel diagnostic and prognostic biomarker in acute ischemic stroke. Aging Dis 7: 614-622, 2016.

18. Kim JT, Park MS, Choi KH, Cho KH, Kim BJ, Han MK, Park TH, Park SS, Lee KB, Lee BC, et al: Different antiplatelet strategies in patients with new ischemic stroke while taking aspirin. Stroke 47: 128-134, 2016

19. Yilmaz AB, Gokhan S, Sener A and Erel O: Analysis of Neutrophil/Lymphocyte ratio and Thiol/Disulfide homeostasis parameters in patients admitted to the emergency department with ischemic stroke. Pak J Med Sci 34: 1418-1423, 2018.

20. Pignatelli P, Carnevale R, Pastori D, Cangemi R, Napoleone L, Bartimoccia S, Nocella C, Basili S and Violi F: Immediate antioxidant and antiplatelet effect of atorvastatin via inhibition of Nox2. Circulation 126: 92-103, 2012.

21. Jauch EC, Saver JL, Adams HP Jr, Bruno A, Connors JJ, Demaerschalk BM, Khatri P, McMullan PW Jr, Qureshi AI, Rosenfield K, et al; American Heart Association Stroke Council; Council on Cardiovascular Nursing; Council on Peripheral Vascular Disease; Council on Clinical Cardiology: Guidelines for the early management of patients with acute ischemic stroke: A guideline for healthcare professionals from the American Heart Association/American Stroke Association. Stroke 44: 870-947, 2013.
22. Hao Y, Qi Z, Ding Y, Yu X, Pang L and Zhao T: Effect of interventional therapy on IL-1 $\beta$, IL-6, and neutrophil-lymphocyte ratio (NLR) levels and outcomes in patients with ischemic cerebrovascular disease. Med Sci Monit 25: 610-617, 2019.

23. Yang Y, Liu H, Zhang H, Ye Q, Wang J, Yang B, Mao L, Zhu W, Leak RK, Xiao B, et al: ST2/IL-33-dependent microglial response limits acute ischemic brain injury. J Neurosci 37: 4692-4704, 2017.

24. Panahi M, Papanikolaou A, Torabi A, Zhang JG, Khan H, Vazir A, Hasham MG, Cleland JGF, Rosenthal NA, Harding SE, et al: Immunomodulatory interventions in myocardial infarction and heart failure: A systematic review of clinical trials and metaanalysis of IL-1 inhibition. Cardiovasc Res 114: 1445-1461, 2018

25. Galun D, Bogdanovic A, Djokic Kovac J, Bulajic P, Loncar Z and Zuvela M: Preoperative neutrophil-to-lymphocyte ratio as a prognostic predictor after curative-intent surgery for hepatocellular carcinoma: Experience from a developing country. Cancer Manag Res 10: 977-988, 2018

26. Dhanesha N, Prakash P, Doddapattar P, Khanna I, Pollpeter MJ, Nayak MK, Staber JM and Chauhan AK: Endothelial cell-derived von Willebrand factor is the major determinant that mediates von Willebrand factor-dependent acute ischemic stroke by promoting postischemic thrombo-inflammation. Arterioscler Thromb Vasc Biol 36: 1829-1837, 2016.

27. Vaz HA, Guimaraes RB and Dutra O: Challenges in highsensitive troponin assay interpretation for intensive therapy. Rev Bras Ter Intensiva 31: 93-105, 2019 (In Portuguese).

28. Zhang J, Zhang J, Sun H, Ming T, Liu X, Cong Y, Li F and Li Z: Association between platelet function and recurrent ischemic vascular events after TIA and minor stroke. Int J Clin Pharmacol Ther 55: 789-797, 2017.

29. Yi X, Han Z, Wang C, Zhou Q and Lin J: Statin and aspirin pretreatment are associated with lower neurological deterioration and platelet activity in patients with acute ischemic stroke. J Stroke Cerebrovasc Dis 26: 352-359, 2017.

30. Furlanis G, Ajčević M, Stragapede L, Lugnan C, Ridolfi M, Caruso P, Naccarato M, Ukmar M and Manganotti P: Ischemic volume and neurological deficit: Correlation of computed tomography perfusion with the National Institutes of Health Stroke Scale Score in acute ischemic stroke. J Stroke Cerebrovasc Dis 27: 2200-2207, 2018.

This work is licensed under a Creative Commons Attribution-NonCommercial-NoDerivatives 4.0 International (CC BY-NC-ND 4.0) License. 